\title{
Capsule Commentary on Melzer et al., Predictors of Pharmacotherapy for Tobacco Use among Veterans Admitted for COPD: The Role of Disparities and Tobacco Control Processes
}

\author{
Kristin Berg, MD, MS \\ Center for Tobacco Research and Intervention, Department of Medicine, University of Wisconsin School of Medicine and Public Health, Madison, \\ WI, USA.
}

J Gen Intern Med 31(6):648

DOI: $10.1007 / \mathrm{s} 11606-016-3663-9$

c) Society of General Internal Medicine 2016

$\mathrm{T}$ his study by Melzer et al. ${ }^{1}$ examined factors related to the receipt of tobacco cessation medications for a cohort of veterans admitted for COPD. Factors examined included patient-level characteristics as well as inpatient tobacco control measures. The authors demonstrated the stark gap in overall prescription of cessation medications: only $16.9 \%$ of these high-risk patients received a tobacco cessation medication at discharge. They further demonstrated the continued disparities in who receives cessation medications. The authors showed that these patients tended to be younger, white, without significant psychiatric or medical comorbidities, and with fewer markers of COPD severity. Effectively, the most high-risk of this already risky cohort were not receiving the medications they needed.

Finally, the authors suggested a gap in what patients are being prescribed. The 2008 Clinical Practice Guideline on treating tobacco use recommended the consideration of combination cessation therapy, ${ }^{2}$ whereas Melzer et al. demonstrated that monotherapy with the nicotine patch was still the mainstay of inpatient treatment. This demonstrates a lingering uncertainty regarding the best approach to treatment, echoed by the results shown in Baker et al.'s randomized trial comparing varenicline, combination nicotine replacement, and the nicotine patch. ${ }^{3}$

This paper is a call to providers, administrators, and researchers that progress is desperately needed. With such clear adverse outcomes, tobacco use must be targeted and treated effectively and efficiently. These results raise multiple questions as to where we are failing our patients. Reports published nearly 10 years ago demonstrated low rates of counseling and medication prescriptions, with similar disparities. ${ }^{4,5}$ Could Melzer et al. have demonstrated improvements over time given their use of data spanning 2005 through 2012? Or, does this reflect frustration on the part of providers and patients based on limited success rates, leading to a lack of counseling or a lack of patient uptake? Perhaps, as Melzer raised, a sense of futility or lack of time on the part of providers? Or, are we lacking standardized procedures to ensure that the positive effects of inpatient tobacco control measures, which were seen in this article, are translated to outpatient prescriptions? Regardless, this important paper continues to drive home the difficult question of how we can most successfully help our patients in need.

Corresponding Author: Kristin Berg, MD, MS; Center for Tobacco Research and Intervention, Department of MedicineUniversity of Wisconsin School of Medicine and Public Health, Madison, WI, USA (e-mail: kberg@medicine.wisc.edu).

\section{Compliance with Ethical Standards:}

Conflict of Interest: The author has no conflict with any of the material in the original article, but collaborates with some of the authors cited in this commentary.

\section{REFERENCES}

1. Melzer AC., et al. Predictors of pharmacotherapy for tobacco use among Veterans admitted for COPD: the role of disparities and tobacco control processes. J Gen Intern Med. 2016; doi: 10.1007/s11606-016-3623-4.

2. Fiore MC, Jaén CR, Baker TB, et al. Treating Tobacco Use and Dependence: 2008 Update. Clinical Practice Guideline. Rockville, MD: U.S. Department of Health and Human Services. Public Health Service; 2008.

3. Baker TB, et al. Effects of nicotine patch vs varenicline vs combination nicotine replacement therapy on smoking cessation at 26 weeks: a randomized clinical trial. JAMA. 2016;315(4):371-9.

4. Browning $\mathbf{K K}$, et al. Socioeconomic disparity in provider-delivered assistance to quit smoking. Nicotine Tob Res. 2008;10(1):55-61.

5. Steinberg MB, et al. Gender and age disparities for smoking-cessation treatment. Am J Prev Med. 2006;30(5):405-12.

Published online March 10, 2016 